\title{
Increased Adverse Skin Reactions Among Healthcare Workers During COVID-19 Outbreak
}

\author{
(D) Tuğba Özkök Akbulut, (1) Tuğba Atcı, (1) Nazlı Caf, (1) Hülya Süslü
}

University of Health Sciences Turkey, Istanbul Haseki Training and Research Hospital, Clinic of Dermatology, Istanbul, Turkey

\section{ABSTRACT}

Background: The personal protective equipment (PPE) and frequent hand hygiene procedures needed during the Coronavirus disease-2019 outbreak impair skin integrity in healthcare workers (HCWs). We aimed to evaluate the prevalence and risk factors of adverse skin reactions related to infection-prevention measures among HCWs

Materials and Methods: A questionnaire survey was administered to evaluate the duration of PPE, disinfectant, and moisturizing agent use, as well as handwashing frequency among our hospital's HCWs.

Results: The questionnaire was completed by $702 \mathrm{HCWs}$ with a mean age of $34.8 \pm 9.8$ years-old. Adverse skin reactions were reported by $79.5 \%$ of our participants. Hands (63.5\%) and face (48.9\%) were the most commonly affected areas. Female sex, being a doctor/nurse, having a history of underlying chronic dermatoses, and PPE usage more than six hours per day were increased the risk of adverse skin reactions. Handwashing more than 10 times/day and moisturizing less than 5 times/day were also related to increased adverse skin reactions. In HCWs, wearing more than one mask was associated with pressure-induced skin changes on the face and triggering herpes labialis.

Conclusion: Hand hygiene-associated dermatitis is triggered by frequent handwashing and less moisturizing among HCWs. Surgical masks may also be just as responsible as N95 masks for causing facial skin damage.

Keywords: COVID-19, Hand disinfection, Personal protective equipment, Healthcare workers

\section{Introduction}

Since the Coronavirus disease-19 (COVID-19) pandemic worldwide, healthcare workers (HCWs) have had to use personal protective equipment (PPE) for long hours and to pay more attention to hand hygiene by frequent hand washing and use of alcohol-based disinfectants. This has made HCWs susceptible to skin damage as a result of infection-prevention measures [1]. In this study, a questionnaire survey was conducted that included questions about the occurrence of skin reactions and the frequency or duration of conducting infection-prevention measures. The goal was to evaluate the prevalence, clinical characteristics, and risk factors of skin reactions among Turkish HCWs during the COVID-19 outbreak. Our results were also compared with similar studies reported worldwide in the literature $[2,3,4,5,6]$. The findings of our study, accompanied by data from the literature review, will help to determine whether infection-prevention measures pose important occupational health risks in HCWs during the COVID-19 pandemic.

\section{Materials and Methods}

This survey was conducted in a tertiary healthcare center by the distribution of a cross-sectional questionnaire that asked about the duration of the use of PPE and gloves, the frequency of handwashing, the use of alcohol-based disinfectants, and hand 
cream application. Participants in this study were medical doctors, nurses, and other healthcare professionals. Demographics, adverse skin reactions, and sites of lesions were recorded. Univariate and multivariate analyses were used to evaluate associations between adverse skin reactions and the following parameters: age, sex, occupation, duration of exposure to PPE, layers and types of gloves, frequency of handwashing, and hand cream application.

\section{Statistical Analysis}

The Statistical Package for the Social Sciences version 22 was used for analysis of the data. The independent t-test, chi-square and Fisher Exact tests were performed to compare the groups. Quantitative data are expressed in the tables as mean \pm standard deviation values. Categorical data are presented as numerical values (n) and percentages (\%). Multivariate analysis was performed using logistic regression analysis from the parameters that were significant in univariate analysis. Data were analyzed at a 95\% confidence level, and a $\mathrm{p}$ value of $<0.05$ was considered statistically significant. All participants signed an informed consent form before the questionnaire survey. The study was approved by the Ethics Committee of Haseki Istanbul Training and Research Hospital and was carried out in accordance with the Declaration of Helsinki.

\section{Results}

The questionnaire was completed by $702 \mathrm{HCWs}$ with a mean age of $34.8 \pm 9.8$ years; 400 (57\%) were women. Among the respondents, $30.8 \%$ were medical doctors, $35.2 \%$ were nurses, and the rest were other HCWs (34\%). A total of 558 (79.5\%) respondents had adverse skin reactions (Table 1). The hands (63.5\%) and face (48.9\%) were the most commonly affected areas.

The univariate analysis revealed that sex, occupation, underlying chronic dermatoses, and duration of exposure to PPE were significantly associated with an adverse skin reaction (Table 1). The multivariate analysis, female sex demonstrated that being a medical doctor/nurse, having a history of underlying chronic dermatoses, and experiencing a duration of exposure to PPE of more than six hours per day were associated with an increased risk of adverse skin reactions (Table 1).

Most of the HCWs washed their hands and/or used disinfectants more than 10 times per day (78.1\% and 66.2\%, respectively); however, only $28.2 \%$ applied hand cream more than 5 times per day. The univariate analysis indicated a significant association between adverse skin reactions on the hands and the frequency of hand washing, the number of moisturizing applications per day and the number of gloves worn (Table 2). The multivariate analysis revealed that adverse skin reactions on the hands were also associated with hand washing more than 10 times per day and with hand moisturizing less than 5 times per day (Table 2).

Adverse skin reactions noted on the face were pressure-induced skin changes (54.3\%), the triggering (28.8\%) and exacerbation (27.8\%) of acne vulgaris and/or acne rosacea, and the triggering of herpes labialis (22.8\%) (Table 3). Wearing more than one mask layer was associated with pressure-induced skin changes and exacerbation

Table 1. Analysis of the risk factors for development of adverse skin reactions

\begin{tabular}{|c|c|c|c|c|c|}
\hline \multirow{2}{*}{ Variable } & \multicolumn{2}{|c|}{ Adverse skin reaction } & \multirow{2}{*}{$\begin{array}{l}\text { Univariate analysis } \\
p \text { value }\end{array}$} & \multicolumn{2}{|c|}{ Multivariate analysis } \\
\hline & Yes $(n=558)(\%)$ & No $(n=144)(\%)$ & & OR $(95 \% \mathrm{Cl})$ & $p$ value \\
\hline Age (years, mean $\pm S D$ ) & $34.5 \pm 9.7$ & $36.0 \pm 9.9$ & 0.060 & & \\
\hline - Male $(n=295)$ & 67.1 & 32.9 & \multirow{2}{*}{0.001} & \multirow{2}{*}{$3.28(2.18-4.93)$} & \multirow{2}{*}{0.001} \\
\hline - Female $(n=400)$ & 88.75 & 11.25 & & & \\
\hline - COVID-19 related $(n=420)$ & 80.9 & 19.1 & \multirow{2}{*}{0.429} & & \\
\hline - Other $(n=282)$ & 77.3 & 22.7 & & & \\
\hline \multicolumn{6}{|l|}{ Occupation } \\
\hline - Medical doctor/nurse $(n=469)$ & 84 & 16 & \multirow{2}{*}{0.001} & \multirow{2}{*}{$1.81(1.20-2.72)$} & \multirow[b]{2}{*}{0.004} \\
\hline - Other medical staff $(n=230)$ & 70.4 & 29.6 & & & \\
\hline \multicolumn{6}{|l|}{ Underlying chronic dermatoses } \\
\hline- No $(n=471)$ & 63.1 & 36.9 & 0.001 & $2.38(1.47-3.86)$ & 0.001 \\
\hline \multicolumn{6}{|l|}{ Duration of PPE per day } \\
\hline$<6$ hours $(n=321)$ & 74.1 & 25.9 & \multirow{2}{*}{0.001} & \multirow{2}{*}{$1.93(1.29-2.88)$} & \multirow{2}{*}{0.001} \\
\hline$\geq 6$ hours $(n=380)$ & 84 & 16 & & & \\
\hline
\end{tabular}


of acne vulgaris and/or acne rosacea, whereas triggering of acne vulgaris and/or acne rosacea was more commonly reported by HCWs who wore a single mask layer (Table 3). In addition, triggering of herpes labialis was more common in our study in HCWs who wore only a single surgical mask layer. No significant relationship was found between the eye protection method and adverse skin reactions on the face in our study (Table 3).

\section{Discussion}

Skin damage due to by infection-prevention measures among HCWs has recently been reported in various countries all over the world $[2,3,4,5,6]$. Our study provides awareness about the risk factors and prevalence for adverse skin reactions associated with infection-prevention measures during the COVID-19 pandemic in Turkey and can be generalized worldwide based on our literature review.

Adverse skin reactions were reported by $79.5 \%$ of our respondents, consistent with results of other studies [2,7]. Sex is known to be a risk factor for some dermatological diseases, and women were found to have an especially higher risk for dermatological complaints during the pandemic in our study, in agreement with some of the previous studies $[2,4,5,6]$. A lower threshold for reporting adverse skin reactions might be related with the higher prevalence of skin symptoms among women.
Skin barrier dysfunction and potential disorder of the skin microbiota of HCWs with underlying chronic dermatoses might be related to observing more common adverse skin reactions in these subjects [5]. The risk of adverse skin reactions in our study was higher for HCWs wearing PPE for more than six hours than for those exposed for less time, in line with previous reports $[5,7,8]$. Interestingly, the working area of the HCWs was not related to the development of adverse skin reactions in our study. A previous study that compared occupational hand eczema between workers in a surgical unit and healthcare professionals in the COVID-19 intensive care unit revealed a significant increase in the development of acute hand dermatitis among all participants, regardless of direct contact with COVID-19 patients, in agreement with our findings [9].

In our study, the hands (63.5\%) were the most commonly affected body part in our HCWs during the pandemic. Similar to previous reports, more frequent (>10 times per day) hand washing, coupled with less frequent hand moisturizing (less than 5 times per day) increased the risk of hand skin damage in our study [4,5,7]. A previous evaluation of dermatological complaints among HCWs found that xerosis and eczema on the hands was increased by 2.44 and 3.57 times, respectively, while hand washing 10 times a day with a hand washing time longer than 10 seconds increased the risk of eczema 5.44 times [4]. The frequency of disinfectant application was not a statistically significant risk factor for hand skin damage in

Table 2. Analysis of risk factors for development of adverse skin reaction on hands

\begin{tabular}{|c|c|c|c|c|c|}
\hline \multirow{2}{*}{ Variable, $(n=)$} & \multicolumn{2}{|c|}{ Adverse skin reaction (hand) } & \multirow{2}{*}{$\begin{array}{l}\text { Univariate analysis } \\
\mathbf{p} \text { value }\end{array}$} & \multicolumn{2}{|c|}{ Multivariate analysis } \\
\hline & Yes $(n=446)(\%)$ & No $(n=256)(\%)$ & & OR $(95 \% \mathrm{Cl})$ & $\mathrm{p}$ value \\
\hline \multicolumn{6}{|c|}{ Frequency of hand washing per day } \\
\hline$\geq 10(n=548)$ & 68.1 & 31.9 & 0.001 & $2.29(1.56-3.35)$ & 0.001 \\
\hline \multicolumn{6}{|c|}{ Frequency of disinfectant use per day } \\
\hline$\geq 10(n=465)$ & 65.2 & 34.8 & 0.209 & & \\
\hline \multicolumn{6}{|c|}{ Frequency of hand moisturing per day } \\
\hline$<5(n=504)$ & 65.7 & 34.3 & \multirow{2}{*}{0.001} & \multirow{2}{*}{$1.57(1.11-2.23)$} & \multirow{2}{*}{0.011} \\
\hline$\geq 5(n=198)$ & 58.1 & 41.9 & & & \\
\hline \multicolumn{6}{|l|}{ Features of gloves } \\
\hline - Latex $(n=605)$ & 63.5 & 36.5 & \multirow{2}{*}{0.925} & & \\
\hline - Nitril $(n=90)$ & 64.4 & 35.6 & & & \\
\hline \multicolumn{6}{|l|}{ Layers of gloves } \\
\hline One $(n=489)$ & 59.9 & 40.1 & \multirow{2}{*}{0.006} & \multirow{2}{*}{$0.47(0.08-2.49)$} & \multirow{2}{*}{0.088} \\
\hline More than one $(n=207)$ & 72.5 & 27.5 & & & \\
\hline
\end{tabular}


our study. Interestingly, previous studies suggested the application of alcohol-based disinfectants instead of soaps for hand hygiene, due to the high antimicrobial effect and low risk of skin reactions, supporting our finding regarding disinfectants $[1,10,11]$.

The long-term use of gloves has also been reported to increase the risk of xerosis and dermatitis on the hands [12]. However, another study also indicated a considerably increased risk even with shortterm glove use for 1 to 2 hours [4]. The virus that causes COVID-19 can exist for several hours on used PPE, so double gloving is recommended to reduce the risks of viral contamination during PPE removal [2,13]. However, wearing more than one layer of gloves increased the risk of hand skin damage in our study. Hypoallergenic gloves, such as nitrile and vinyl gloves, have been recommended for the prevention of hand dermatitis among HCWs, [11] but our findings did not show any statistically significant difference between latex and nitrile gloves.

Previously, various adverse skin reactions were reported in more than a third of HCWs who wore N95 masks [12]. In our study, 28.8\% and $27.8 \%$ of our patients reported triggering and exacerbation of acne vulgaris and acne rosacea, respectively, due to masks, and the presence of pressure-induced skin changes on the face was noted in $54.3 \%$ of the HCWs. The $\mathrm{N} 95$ masks have been reported to cause more adverse skin reactions on the face than surgical masks due to higher air impermeability and more local pressure [14]. However, the findings of our study did not support this difference, as the occurrence of adverse skin reactions did not differ between N95 and surgical masks in our study. The exception was the triggering of herpes labialis, which was more common in HCWs who wore surgical masks.

Some studies evaluating skin reactions due to N95 and surgical mask wear among HCWs have indicated that N95 masks are associated with more reactions than other medical masks, in contrast with the results of our study $[5,14]$. Interestingly, another study evaluated several skin parameters, including skin hydration, transepidermal water loss, erythema, $\mathrm{pH}$, and sebum secretion, on areas covered by the N95 and medical masks versus uncovered skin. At 2 and 4 hours of wear, and at 0.5 and 1 hour after taking off the masks, no significant differences were found between the N95 and medical masks for any of the skin parameters [14]. These previous findings may explain why no significant differences were noted between N95 and surgical masks regarding pressure-induced skin changes or triggering and exacerbation of acne vulgaris and/ or acne rosacea in our study. A previous self-questionnaire study evaluating face mask-induced itch among members of the general public also showed no significant difference between in wearers using a surgical mask, cloth mask, or $\mathrm{N} 95$ mask, in agreement with our study [15]. In some previous studies, HCWs who wore surgical masks, paper masks, and cloth masks did not report any adverse skin reactions $[6,12]$.

A fivefold increase in acne complaints was previously reported for the use of any mask type [4]. The flare-up of acne caused by long-time mask-wearing during the COVID-19 pandemic has been reported among the general population and was associated primarily with medical masks [16]. Friction or bursting of comedones, occlusion of pilosebaceous ducts, and formation of a wet environment conducive to bacterial proliferation may be responsible for the acne complaints related to mask use [11]. Another study evaluating the PPE induced facial dermatoses in HCWs found that goggles were the most common equipment among all PPE to cause any of the dermatoses, with N95 masks and face shields being the next major causes [17]. Conversely, in our study, no significant relationship was noted between eye protection methods and adverse skin reactions on the face.

\section{Study Limitations}

Our study has some limitations. In our study, there may be an answer bias depending on the answers given by the healthcare professionals themselves. This is a self-administered questionnaire

Table 3. Analysis of risk factors for development of adverse skin reaction on face

\begin{tabular}{|c|c|c|c|c|}
\hline Variables & $\begin{array}{l}\text { Pressure-induced skin } \\
\text { changes }(n=381)\end{array}$ & $\begin{array}{l}\text { Triggering of acne vulgaris } \\
\text { and/or acne rosacea }(n=202)\end{array}$ & $\begin{array}{l}\text { Exacerbation of acne vulgaris } \\
\text { and/or acne rosacea }(n=195)\end{array}$ & $\begin{array}{l}\text { Triggering of herpes } \\
\text { labialis }(n=160)\end{array}$ \\
\hline $\begin{array}{l}\text { Layers of mask } \\
\text { - One }(n=489)\end{array}$ & $\begin{array}{l}\mathbf{p}=\mathbf{0 . 0 4 6} \\
200 / 489(40.9 \%)\end{array}$ & $\begin{array}{l}\mathbf{p}=\mathbf{0 . 0 1 2} \\
98 / 489(20 \%)\end{array}$ & $\begin{array}{l}\mathbf{p}=\mathbf{0 . 0 2 5} \\
96 / 489(19.6 \%)\end{array}$ & $\begin{array}{l}\mathbf{p}=\mathbf{0 . 1 2 2} \\
98 / 489(20 \%)\end{array}$ \\
\hline - More than one $(n=207)$ & 181/207 (87.4\%) & $10 / 207(4.8 \%)$ & 99/207 (47.8\%) & $62 / 207(30 \%)$ \\
\hline $\begin{array}{l}\text { Features of mask } \\
\text { - N95 }(n=109) \\
\text { - Surgical }(n=346)\end{array}$ & $\begin{array}{l}p=0.458 \\
58(53.2 \%) \\
170(49.1 \%)\end{array}$ & $\begin{array}{l}\mathbf{p}=\mathbf{0 . 2 6 0} \\
31(28.4 \%) \\
80(23.1 \%)\end{array}$ & $\begin{array}{l}\mathbf{p}=\mathbf{0 . 4 2 7} \\
30(27.5 \%) \\
83(24 \%)\end{array}$ & $\begin{array}{l}p=0.024 \\
15(13.8 \%) \\
83(24 \%)\end{array}$ \\
\hline $\begin{array}{l}\text { Eye protection } \\
\text { - None }(n=191)\end{array}$ & $\begin{array}{l}\mathbf{p}=\mathbf{0 . 1 3 3} \\
96(50.3 \%)\end{array}$ & $\begin{array}{l}\mathbf{p}=\mathbf{0 . 1 8 1} \\
55(28.8 \%)\end{array}$ & $\begin{array}{l}\mathbf{p}=\mathbf{0 . 3 8 7} \\
48(25.1 \%)\end{array}$ & $\begin{array}{l}\mathbf{p}=\mathbf{0 . 1 0 6} \\
48(25.1 \%)\end{array}$ \\
\hline - Goggles (n=151) & $83(55 \%)$ & $35(23.2 \%)$ & $37(24.5 \%)$ & $32(21.2 \%)$ \\
\hline - Face shields $(n=207)$ & $107(51.7 \%)$ & $59(28.5 \%)$ & $65(31.4 \%)$ & 37 (17.9\%) \\
\hline - Both $(n=153)$ & $95(62.1 \%)$ & $53(34.6 \%)$ & $45(29.4 \%)$ & $43(28.1 \%)$ \\
\hline
\end{tabular}


study analyzing the adverse skin reactions as felt by respondents, rather than as evaluated by dermatologists.

\section{Conclusion}

Our study demonstrates that HCWs, and especially females with a history of underlying chronic dermatosis and with a longer exposure time to PPE wear, are particularly affected by adverse skin reactions in Turkey. Hand skin damage is triggered primarily by frequent hand washing, but less frequently by hand moisturizing. The findings in our study also suggest that surgical masks may be just as responsible as N95 masks for causing facial skin damage. This information may be useful for interventions intended to minimize the dermatological complaints of HCWs triggered by infection-prevention measures that impact their performance and quality of life.

\section{Ethics}

Ethics Committee Approval: The study was approved by the Ethics Committee of Istanbul Haseki Training and Research Hospital (number: 127, date: 24/06/2020).

Informed Consent: This study was performed followed the Declaration of Helsinki Principles and formal consent was taken from participants before the survey.

Peer-review: Externally and internally peer-reviewed.

\section{Authorship Contributions}

Surgical and Medical Practices: T.Ö.A., T.A., N.C., H.S., Concept: T.Ö.A. Design: T.Ö.A., T.A., Data Collection or Processing: T.Ö.A., N.C., H.S., Analysis or Interpretation: T.Ö.A., T.A., Literature Search: T.Ö.A., T.A., N.C., H.S., Writing: T.Ö.A., T.A.

Conflict of Interest: No conflict of interest was declared by the authors.

Financial Disclosure: The authors declared that this study received no financial support.

\section{References}

1. Yan Y, Chen H, Chen L, Cheng B, Diao P, Dong L, Gao X, Gu H, He L, Ji C, Jin H, Lai W, Lei T, Li L, Li L, Li R, Liu D, Liu W, Lu Q, Shi Y, Song J, Tao J, Wang B, Wang G, Wu Y, Xiang L, Xie J, Xu J, Yao Z, Zhang F, Zhang J, Zhong S, Li H, Li H. Consensus of Chinese experts on protection of skin and mucous membrane barrier for health-care workers fighting against coronavirus disease 2019. Dermatol Ther 2020;33:e13310.

2. Lin P, Zhu S, Huang Y, Li L, Tao J, Lei T, Song J, Liu D, Chen L, Shi Y, Jiang S, Liu Q, Xie J, Chen H, Duan Y, Xia Y, Zhou Y, Mei Y, Zhou X, Wu J, Fang M, Meng Z, Li $\mathrm{H}$. Adverse skin reactions among healthcare workers during the coronavirus disease 2019 outbreak: a survey in Wuhan and its surrounding regions. Br J Dermatol 2020;183:190-192.

3. Gheisari M, Araghi F, Moravvej H, Tabary M, Dadkhahfar S. Skin reactions to non-glove personal protective equipment: an emerging issue in the COVID-19 pandemic. J Eur Acad Dermatol Venereol 2020;34:e297-e298.

4. Metin N, Turan Ç, Utlu Z. Changes in dermatological complaints among healthcare professionals during the COVID-19 outbreak in Turkey. Acta Dermatovenerol Alp Pannonica Adriat 2020;29:115-122.

5. Zuo Y, Hua W, Luo Y, Li L. Skin reactions of N95 masks and medial masks among health-care personnel: A self-report questionnaire survey in China. Contact Dermatitis 2020;83:145-147.

6. Hu K, Fan J, Li X, Gou X, Li X, Zhou X. The adverse skin reactions of health care workers using personal protective equipment for COVID-19. Medicine (Baltimore) 2020;99:e20603.

7. Lan J, Song Z, Miao X, Li H, Li Y, Dong L, Yang J, An X, Zhang Y, Yang L, Zhou $\mathrm{N}$, Yang L, Li J, Cao J, Wang J, Tao J. Skin damage among health care workers managing coronavirus disease-2019. J Am Acad Dermatol 2020;82:12151216.

8. Pei S, Xue Y, Zhao S, Alexander N, Mohamad G, Chen X, Yin M. Occupational skin conditions on the front line: a survey among 484 Chinese healthcare professionals caring for Covid-19 patients. J Eur Acad Dermatol Venereol 2020;34:e354-e357.

9. Guertler A, Moellhoff N, Schenck TL, Hagen CS, Kendziora B, Giunta RE, French LE, Reinholz M. Onset of occupational hand eczema among healthcare workers during the SARS-CoV-2 pandemic: Comparing a single surgical site with a COVID-19 intensive care unit. Contact Dermatitis 2020;83:108-114.

10. Larson E. Skin hygiene and infection prevention: more of the same or different approaches? Clin Infect Dis 1999;29:1287-1294.

11. Araghi F, Tabary M, Gheisari M, Abdollahimajd F, Dadkhahfar S. Hand Hygiene Among Health Care Workers During COVID-19 Pandemic: Challenges and Recommendations. Dermatitis 2020;31:233-237.

12. Foo CC, Goon AT, Leow YH, Goh CL. Adverse skin reactions to personal protective equipment against severe acute respiratory syndrome--a descriptive study in Singapore. Contact Dermatitis 2006;55:291-294.

13. Patruno C, Fabbrocini G, Stingeni L, Napolitano M. The role of occupational dermatology in the COVID-19 outbreak. Contact Dermatitis 2020;83:174-175.

14. Hua W, Zuo Y, Wan R, Xiong L, Tang J, Zou L, Shu X, Li L. Short-term skin reactions following use of N95 respirators and medical masks. Contact Dermatitis 2020;83:115-121.

15. Szepietowski JC, Matusiak Ł, Szepietowska M, Krajewski PK, Białynicki-Birula R. Face Mask-induced Itch: A Self-questionnaire Study of 2,315 Responders During the COVID-19 Pandemic. Acta Derm Venereol 2020;100:adv00152.

16. Han C, Shi J, Chen Y, Zhang Z. Increased flare of acne caused by long-time mask wearing during COVID-19 pandemic among general population. Dermatol Ther 2020;33:e13704.

17. Singh M, Pawar M, Bothra A, Maheshwari A, Dubey V, Tiwari A, Kelati A. Personal protective equipment induced facial dermatoses in healthcare workers managing Coronavirus disease 2019. J Eur Acad Dermatol Venereol 2020;34:e378-e380. 